\title{
Perceived Parental Control in Chinese Adolescents in Hong Kong: A Three-Year Longitudinal Study
}

\author{
Daniel T.L. Shek \\ Centre for Quality of Life, Hong Kong Institute of Asia-Pacific Studies, The Chinese University of Hong Kong, China
}

\begin{abstract}
Utilizing a longitudinal research design, perceived parental control in 2,559 Chinese adolescents over three consecutive years was examined by measures of indigenous Chinese parental control concepts (Chinese Paternal Control Scale: CPCS; Chinese Maternal Control Scale: CMCS). The relationships between CPCS and CMCS and measures of parental control and parent-child relational qualities were also investigated. Although Chinese parents had high expectations about their children, they were not strict in parental discipline. The CPCS and CMCS scores were significantly correlated with measures of parental control and perceived parental endorsement of traditional Chinese parenting beliefs in early adolescent years. While CPCS and CMCS had weak concurrent and prospective relationships with parent-child relational qualities measures, the observed relationships were moderated by parental psychological control. The theoretical and practical implications of the findings are discussed.
\end{abstract}

Keywords: Chinese adolescents, parental control, behavioral control, psychological control, parent-child relational qualities.

Parents usually exercise control over the behavior of their children in the socialization process. In different models of parenting (e.g., Becker, 1964; Schaefer, 1959), control versus autonomy was used as a dimension to describe parenting characteristics. Besides, different types of parental control (Barber, 1996, 2002), such as psychological control and behavioral control have been proposed. According to Smetana and Daddis (2002), psychological control refers to "parents" attempt to control the child's activities in ways that negatively affect the child's psychological world and thereby undermines the child's psychological development" (p.563) whereas behavioral control refers to "rules, regulations and restrictions that parents have for their children" (p.563).

As far as assessment of parental control is concerned, most of the existing measures of parental control, including indicators of psychological control and behavioral control (e.g., parental knowledge, parental expectations, parental monitoring, and parental discipline), are developed in the West based on concepts in psychology and allied professions (Shek, 2007). Obviously, one should ask whether it is appropriate to use translated Western measures in non-Western contexts. For those who believe in universality of parenting processes across cultures, they would argue that there is no problem in using translated Western measures in nonWestern contexts. In fact, this is the case in existing Chinese parenting research where Western concepts and translated measures of parenting are commonly used in the existing studies. On the other hand, there are researchers who argue that Western concepts of parenting may not be applicable to Chinese people and indigenous Chinese concepts of parenting should be used (e.g., Chao, 1994). In view of the fact that Chinese people constitute roughly one-fifth of the world's

\footnotetext{
*Address correspondence to author at the Centre for Quality of Life, Hong Kong Institute of Asia-Pacific Studies, The Chinese University of Hong Kong, China; E-mail: danielshek@cuhk.edu.hk
}

population, more research on parental control in different Chinese contexts is badly needed (Shek, 2006b).

\section{PARENTAL CONTROL IN THE TRADITIONAL CHINESE CULTURE}

A survey of the literature shows that there were several features intrinsic to traditional Chinese families. First, under the Confucian, Buddhist and Taoist thoughts, harmony in the family was encouraged and conflict among family members was prohibited in traditional Chinese families. As such, children were expected to show good and proper behavior (Shek, 2007). Second, to achieve harmonious social order, behavior of family members was regulated by well-defined duties, obligations and rules. With reference to the "five cardinal relations" ("wu lun"), Chinese children were taught to be submissive to their parents (particularly their fathers) and respect old people. Third, because expression of individual emotions and views would easily create interpersonal tension and conflict, expression of self and emotion was deemphasized in the traditional Chinese culture and there was a strong emphasis of forbearance and patience. Fourth, there was a strong emphasis in the traditional Chinese culture that family members in different generations should live together. In addition, there was a strong emphasis on family teaching ("jia jiao"), such as bringing honor to the family and not to disgrace its good name, and continuation of the family name.

Yang (1981) highlighted several features of traditional Chinese socialization practice. First, children were taught to depend on their parents (dependency training). Second, interpersonal harmony was encouraged whereas interpersonal conflict was discouraged (conformity training). Third, selfinhibition and self-sacrifice were cultivated (self-suppression training). Fourth, self-assertiveness was deemphasized (humility training). Fifth, children were taught to accept fate (contended mentality training). Sixth, deviance was punished severely (punishment orientation). Finally, views of the parents were accorded a higher priority than those of children 
(parent-centeredness). In short, as pointed out by Chao and Tseng (2002), centrality of the family, family interdependence, strict parental control and harsh socialization were emphasized in traditional Chinese parenting.

With specific focus on parental control, Shek (2007) highlighted several features of parental control in the traditional Chinese culture. First, Chinese parental control was characterized by features of psychological control. According to Barber and Harmon (2002), both higher-order attributes (i.e., covert parenting characteristics such as indirect control of the child, intrusiveness, and hostility) as well as lower-order features (i.e., explicit parenting characteristics such as manipulative, constraining, and excessive parental expectations as well as affective punishment) are elements of psychological control. With reference to this conceptualization, features of psychological control can indeed be found in traditional Chinese parenting, such as demand for absolute obedience of the child, emphasis of the faultless nature of parents, unconditional respect for the parents, and intrusiveness of parents in the socialization process.

Second, parents in the traditional Chinese culture had high expectations about their children, particularly the sons. Children were expected to have attributes of maturity ("sheng xing"), obedience ("guai"), filial piety, good virtues, good social behavior, honoring the family name, respect for parent, and honoring senior members in the family. These expectations were summarized by Ho (1986) that "traditionally, great emphasis was placed on obedience, proper conduct, moral training, and acceptance of social obligation, in contrast to the lack of emphasis placed on independence, assertiveness, and creativity" (p. 35-36).

Finally, to ensure that parental expectations were fulfilled, strict and firm discipline particularly via punishment was commonly used, as reflected by the saying of "bang xia chu xiao zi" (a filial son is the product of the rod). A review of the "family handbooks" in the traditional Chinese culture showed that there was a strong emphasis of family rules ("jia gui") and how children should be punished under different circumstances (Shek \& Lai, 2000). In addition, fathers were expected to play the role of teachers to supervise the children to ensure that they behave well, as reflected in the saying of "yang bu jiao, fu zhi guo" (it is the fault of the father if he only raises the child without teaching him). In short, the above discussion suggests that the dominant features of traditional Chinese parental control included features of psychological control (e.g., parental intrusiveness), high parental expectations and harsh discipline. From the perspective of modern parenting science, these features (particularly psychological control and excessive parental expectations) can be regarded as detrimental to parent-child relational qualities.

With gradual modernization and industrialization in different Chinese societies (such as Hong Kong and mainland China), it is reasonable to expect that contemporary parental control attributes are influenced by traditional Chinese values as well as modern Western ideas. Therefore, it would be interesting to explore how Chinese adolescents perceive the characteristics of parental control indexed by traditional Chinese parenting characteristics.

\section{ASSESSMENT OF CHINESE PARENTAL CONTROL}

There are very few published scientific studies on Chinese parental control using indigenous Chinese concepts. To assess Chinese parental control based on indigenous Chinese concepts, Shek (2007) developed the Chinese Paternal Control Scale (CPCS) and Chinese Maternal Control Scale (CMCS). Consistent with the predictions, Shek (2007) showed that parental control indexed by these two scales was more strongly related to parental psychological control, parental expectation and parental discipline than to parental knowledge and parental monitoring. In addition, the relationship between parental control assessed by the indigenous concepts and parent-child relational qualities appeared to be moderated by psychological control. Although Shek's (2007) study was the first published study utilizing indigenously developed measures to assess Chinese parental control and their relationships to parent-child relational qualities, the data were based on a cross-sectional design. Obviously, it would be illuminating if longitudinal data can be collected to illuminate Chinese parental control throughout the early adolescent years.

\section{RESEARCH QUESTIONS AND HYPOTHESES}

Three research questions are addressed in this paper.

Research Question 1: What is the nature of perceived parental control among Chinese adolescents in Hong Kong? Based on the frequency of responses to the items in the CPCS and CMCS, the picture on Chinese paternal and maternal control in the early adolescent years can be clarified. This question is important because related findings in this area are almost non-existent. As this is a descriptive research question, no specific hypothesis was proposed.

Research Question 2: What are the relationships between parental control using indigenous Chinese concepts and parental behavior control, psychological control and parental endorsement of traditional Chinese parenting beliefs? To fully understand the relationship between Chinese parental control and different aspects of parental behavioral control, several aspects of parental behavioral control, including parental knowledge, parental expectation, parental monitoring, and parental discipline were employed.

There are several hypotheses related to this research question. First, as Chinese parental control has the flavor of psychological control and behavioral control, it was expected that Chinese parental control measures would be related to measures of psychological control and behavioral control (Hypothesis 1A). Second, as Chinese parental control concepts were closely related to traditional Chinese beliefs about parenting, it was expected that Chinese parental control measures would have significant relationships with perceived parental endorsement of traditional Chinese beliefs, such as filial piety and harsh punishment (Hypothesis 1B). Finally, as Chinese parental control attributes were more closely related to psychological control, parental expectations and parental discipline (see arguments presented above), it was expected that the CPCS and CMCS would have stronger relationships with psychological control, parental expectations and strict parental discipline than with other parenting attributes, such as parental knowledge and parental monitoring (Hypothesis 1C). This hypothesis is also consistent with the findings of Shek (2007). 
Research Question 3: What are the relationships between Chinese parental control and parent-child relational qualities? To give a more comprehensive picture about this issue, several indices of parent-child relational qualities, including satisfaction with parental control, child's readiness to communicate with the parent, parental trust of the child and child's trust of the parent were used (Shek, 2007). Previous Western studies showed that psychological control was negatively related to parent-child relational quality (e.g. Harakeh et al., 2004), whereas parental behavioral control measures were positively related to parent-child relational quality (Shek \& Lee, 2005). However, as the above discussion suggests that Chinese parental control is a mixture of psychological control, parental expectation and discipline, it was expected that the relationships between the CPCS and CMCS and indicators of parent-child relational qualities would be weaker than the relationships between Western measures of parental control and indicators of parent-child relational qualities. In particular, as parental monitoring and parental knowledge were commonly used as measures of parental control in the West, it was predicted that the correlation coefficients between CPCS (and CMCS) and measures of parent-child relational qualities would be significantly lower than the related correlation coefficients between parental monitoring and parental knowledge and parent-child relational qualities (Hypothesis 2A). However, as psychological control impaired parent-child relationship (Barber, 1996, 2002), it was further expected that Chinese parental control would be more positively related to parent-child relational quality under low parental psychological control condition relative to the high parental psychological control condition. This expectation is in line with the findings of Shek (2007) that psychological control moderated the relationship between Chinese parental control and parent-child relational quality measures (Hypothesis 2B).

\section{METHOD}

\section{Participants and Procedures}

A longitudinal research design with three waves of data (Wave 1, Wave 2 and Wave 3 data) was used to examine perceived parental control and parent-child relational quality in Chinese early adolescents in Hong Kong. At Time 1, 3,017 secondary school students (1331 boys and 1670 girls) participated in the study. They were all Secondary 1 (i.e., Grade 7) students (mean age $=12.65$ years) and were recruited from 16 schools randomly selected from all secondary schools in Hong Kong. The second assessment (Time 2) occurred one year after the first assessment (Time 1) where the students were asked to respond to the same questionnaire used at Time 1. Follow-up data could be obtained from 2,758 students at Time 2. The third assessment occurred one year after the second assessment (Time 3) where the students were asked to respond to the same questionnaire used at Time 1. Follow-up data could be obtained from 2,559 students at Time 3. This group of students, from whom the Time 1, Time 2 and Time 3 data were collected, constituted the longitudinal sample for the study. The Time 1, Time 2 and Time 3 data based on the longitudinal sample were used in the study. Ethical approval from The Chinese University of Hong Kong was obtained prior to data collection.

At each wave of data collection, the purpose of the study was mentioned, and the confidentiality of the data collected was repeatedly emphasized to all of the students in attendance on the day of testing. School consent, parental consent and "passive" student consent were obtained (i.e., the students could freely choose not to participate in the study if they did not wish). The participants completed the questionnaires in class periods with the presence of trained research assistants. All participants responded en masse to all instruments in the questionnaire in a self-administration format. Adequate time was provided for the participants to complete the questionnaire. The questionnaire took roughly 45 to 60 minutes to complete.

\section{Instruments}

To answer the research questions, three groups of measures were used. First, measures of paternal and maternal control based on indigenous Chinese concepts were used. Second, measures of parental behavioral control, psychological control and perceived parental endorsement of traditional Chinese parenting beliefs were used. Finally, measures of perceived parent-child relational qualities were utilized. For all measures, items in the scales were rated on a 4-point scale ranging from 1 (strongly disagree) to 4 (strongly agree).

\section{Assessment of Parental Control Based on Indigenous Chinese Concepts}

Chinese Paternal Control Scale (CPCS) and Chinese Maternal Control Scale (CMCS): For the CPCS, the following items were developed to assess parental control of the fathers: (1) "My father expects me to be mature (sheng xing)." (2) "My father expects me to be obedient ("guai" and "ting hua")." (3) "My father expects me to have good virtues and behavior." (4) "My father expects me to have good behavior so that I will not bring dishonor to the family ("you ru jia sheng")." (5) "My father expects me to have good behavior so that he will not be criticized by others as having no family teaching ("wu jia jiao")." (6) "My father expects me to respect him." (7) "My father is very harsh in his discipline." (8) "My father always teaches me about the ways of dealing with one self and others." (9) "When I do something wrong, my father requires me to have self-reflection." (10) "When I do something wrong, my father teaches me ("jiao xun")." (11) When I don't meet my father's expectation, he urges me ("du cu") to work hard." (12) "My father expects me to interact with older people ("chang bei") with respect and courtesy." The above items were also used to assess control of the mothers by changing the parent's status in the items. The total score of the items in each scale was used as an indicator of the degree of parental control based on Chinese concepts, with a higher score indicating a higher level of Chinese parental control.

\section{Assessment of Perceived Parental Control and Perceived Parental Endorsement of Traditional Chinese Parenting Beliefs}

Paternal Psychological Control Scale (PPSY) and Maternal Psychological Control Scale (MPSY). Based on literature review (Barber, 1996; Smetana \& Daddis, 2002), ten items were developed to form the Paternal Psychological Control Scale (e.g., "My father always wants to change my thoughts"). Identical items with reference to "my mother" were used to assess maternal psychological control that formed the Maternal Psychological Control Scale. A higher 
total scale score indicates a higher level of psychological control. Shek (2006a) showed that these two scales possessed acceptable psychometric properties.

Paternal Knowledge Scale (PKNO) and Maternal Knowledge Scale (MKNO). As parental knowledge has been commonly used to assess parental behavioral control in the Western literature, it is an important indicator of parental behavioral control (Shek, 2005). Based on literature review (e.g., Kerr \& Stattin, 2000), seven items were developed to form the Paternal Knowledge Scale (e.g., "My father clearly knows my situation in my school"). Identical items with reference to "my mother" were used to assess maternal knowledge (Maternal Knowledge Scale). A higher total score in each scale indicates a higher level of parental knowledge of the child's behavior. Based on factor analyses of the perceived parental behavioral control measures, Shek (2005) showed that these two scales had good construct validity.

Paternal Expectation Scale (PEXP) and Maternal Expectation Scale (MEXP). Based on literature review (e.g., Galambos, Barker \& Almeida, 2003), seven items were developed to form the Paternal Expectation Scale (e.g., "My father requires me to have good behavior in school"). Identical items with reference to "my mother" were used to assess maternal expectation (Maternal Expectation Scale). A higher score in each scale indicates a higher level of expectation and requirement of the child's behavior. There are research findings supporting the psychometric properties of the scales (Shek, 2005, 2006c).

Paternal Monitoring Scale (PMON) and Maternal Monitoring Scale (MMON). Based on the existing studies (Pettit, Laird, Dodge, Bates \& Criss, 2001), seven items were developed to form the Paternal Monitoring Scale (e.g., "My father actively understands my situation in school"). Identical items with reference to "my mother" were used to assess maternal monitoring that formed the Maternal Monitoring Scale. A higher score in each scale indicates a higher level of parental monitoring of the child's behavior. There are research findings supporting the psychometric properties of the scales (Shek, 2005, 2006c).

Paternal Discipline Scale (PDIS) and Maternal Discipline Scale (MDIS). Based on a review of the literature, four items were developed to form the Paternal Discipline Scale (e.g., "When I study hard, my father praises me"). Identical items with reference to "my mother" were used to assess maternal discipline that formed the Maternal Discipline Scale. A higher score in each scale indicates a higher level of reasonable parental discipline of the child. There are research findings supporting the psychometric properties of the scales (Shek, 2005, 2006c).

Paternal Parenting Beliefs Scale (PBEF) and Maternal Parenting Beliefs Scale (MBEF): Five items based on the literature (e.g., Shek \& Lai, 2000) were developed to assess the respondent's perception of the traditional parenting beliefs held by the fathers that formed the Paternal Parenting Beliefs Scale (e.g., "my father believes that children must obey their parents under any circumstances"). Identical items were used to assess children's perception of maternal parenting beliefs that formed the Maternal Parenting Beliefs Scale. The total score of the items in each scale was used as an indicator of the perceived level of endorsement of traditional parenting beliefs of the parent, with a higher score indicating a higher level of endorsement of the traditional parenting beliefs.

\section{Assessment of Parent-Child Relational Qualities}

Satisfaction with Paternal Control Scale (SATF) and Satisfaction with Maternal Control Scale (SATM). Based on the literature, four items were developed to form the Satisfaction with Paternal Control Scale (e.g., "I feel that how my father disciplines me is reasonable"). Identical items with reference to "my mother" were used to assess the respondent's satisfaction with maternal control that formed the Satisfaction with Maternal Control Scale. A higher total score of each scale reflects the level of satisfaction of the child regarding parental control. There are research findings supporting the psychometric properties of the scales (Shek, Lee \& Chow, 2006).

Readiness to Communicate with the Father Scale (RCF) and Readiness to Communicate with the Mother Scale $(R C M)$. Two items were developed to assess the respondents' readiness to communicate with the father and formed the Readiness to Communicate with the Father Scale. Identical items with reference to "my mother" were used to assess the children's readiness to share with their mothers that formed the Readiness to Communicate with the Mother Scale. A higher total score in each scale indicates a higher level of readiness of the child to communicate with the parent. There are research findings supporting the psychometric properties of the scales (Shek, Lee \& Chow, 2006).

Paternal Trust of Children Scale (PTRU) and Maternal Trust of Children Scale (MTRU). Five items were developed to assess the respondents' perceptions of their fathers' trust in them that formed the Paternal Trust of Children Scale (e.g., "My father trusts that I will not mix with bad friends"). Identical items with reference to "my mother" were used to assess children's perception of maternal trust about themselves which formed the Maternal Trust of Children Scale. A higher total scale score indicates a higher level of perceived parental trust. There are research findings supporting the psychometric properties of the scales (Shek, Lee \& Chow, 2006).

Children's Trust of Father Scale (TRUF) and Children's Trust of Mother Scale (TRUM). Five items were used to assess the respondents' trust of their fathers that formed the Children's Trust of Father Scale (e.g., "I trust that my father will not cheat me"). Identical items with reference to "my mother" were used to assess the child's perception of their trust of the mothers (Children's Trust of Mother Scale). A higher total scale score indicates a higher level of trust. There are research findings supporting the psychometric properties of the scales (Shek, Lee \& Chow, 2006).

\section{RESULTS}

\section{Reliability of the Measures Used in the Study}

Regarding the measures of Chinese parental control using indigenous Chinese concepts, results showed that the CPCS was internally consistent (alpha $=.85, .87$ and .87 at Grade 7 , Grade 8 and Grade 9, respectively). Similarly, the alpha values associated with the CMCS were respectable $(.84, .85$ and 
Table 1. Values of Coefficient Alpha of the Assessment Tools Used at Grade 7, Grade 8 and Grade 9

\begin{tabular}{|c|c|c|c|c|c|c|c|}
\hline Measure & Grade 7 & Grade 8 & Grade 9 & Measure & Grade 7 & Grade 8 & Grade 9 \\
\hline \hline CPCS & .85 & .87 & .87 & CMCS & .84 & .85 & .86 \\
\hline PPSY & .89 & .90 & .91 & MPSY & .90 & .91 & .91 \\
\hline PKNO & .82 & .84 & .84 & MKNO & .85 & .85 \\
\hline PEXP & .72 & .77 & .76 & MEXP & .71 & .75 & .76 \\
\hline PMON & .82 & .85 & .84 & MMON & .79 & .81 & .81 \\
\hline PDIS & .67 & .71 & .68 & MDIS & .68 & .70 & .71 \\
\hline PBEF & .77 & .81 & .81 & MBEF & .80 & .82 & .82 \\
\hline SATF & .78 & .82 & .82 & SATM & .75 & .80 & .81 \\
\hline RCF & .90 & .93 & .92 & RCM & .79 & .93 & .93 \\
\hline PTRU & .81 & .83 & .83 & MTRU & .81 & .80 \\
\hline TRUF & .77 & .79 & .80 & TRUM & .84 & .77 \\
\hline
\end{tabular}

Note. CPCS: Chinese Paternal Control Scale. PPSY: Paternal Psychological Control Scale. PKNO: Paternal Knowledge Scale. PEXP: Paternal Expectation Scale. PMON: Paternal Monitoring Scale. PDIS: Paternal Discipline Scale. PBEF: Paternal Parenting Beliefs Scale. SATF: Satisfaction with Paternal Control Scale. RCF: Readiness to Communicate with Father Scale. FTRU: Paternal Trust of Children Scale. TRUF: Children's Trust of Father Scale. CMCS: Chinese Maternal Control Scale. MPSY: Maternal Psychological Control Scale. MKNO: Maternal Knowledge Scale. MEXP: Maternal Expectation Scale. MMON: Maternal Monitoring Scale. MDIS: Maternal Discipline Scale. MBEF: Maternal Parenting Beliefs Scale. SATM: Satisfaction with Maternal Control Scale. RCM: Readiness to Communicate with Mother Scale. MTRU: Maternal Trust of Children Scale. TRUM: Children's Trust of Mother Scale.

.85 at Grade 7, Grade 8 and Grade 9, respectively). For the internal consistency of measures of perceived behavioral control (parental knowledge, parental expectation, parental monitoring, parental discipline), psychological control, parental endorsement of traditional Chinese parenting beliefs, and parent-child relational qualities (satisfaction with parental control, readiness to communicate with parents, parental trust of the child, and trust of parents), results in Table 1 showed that they were generally consistent at different time points in early adolescence.

\section{Research Question 1 (Nature of Perceived Parental Con- trol Based on CPCS and CMCS)}

The findings based on the participants' responses to different items of the CPCS at different time points are presented in Table 2. Several interesting observations can be highlighted from the findings. First, although most fathers were perceived to have expectations about their children in terms of maturity (item 1), obedience (item 2), virtues (item 3 ), respect for father (item 6) and respect for older people (item 12), paternal expectations for good behavior for family reasons (items 4 and 5) were not overwhelming. Second, some fathers did not play the role as a teacher (item 8 and item 11). Third, a significant proportion of the children were not requested by their fathers to have self-reflection (item 9). Finally, contrary to the Chinese emphasis of harsh training, only roughly half of the respondents perceived paternal discipline as harsh (item 7). Similar patterns were observed for the responses to the items in the CMCS at different time points.

\section{Research Question 2 (Hypotheses 1A, 1B and 1C)}

Analyses based on the multi-stage Bonferroni procedures (Larzelere \& Mulaik, 1977) showed that the CPCS and CMCS scores were significantly related to measures of parental psychological control and behavioral control (parental knowledge, expectations, monitoring and discipline) and perceived parental endorsement of traditional parenting beliefs, thus supporting Hypothesis $1 \mathrm{~A}$ and Hypothesis 1B (Table 3). Regarding Hypothesis 1C, results showed that the CPCS and CMCS had significantly higher correlation with measures of psychological control, parental expectations, and parental discipline than with parental knowledge and parental monitoring $(\mathrm{p}<.05$ in all cases in Table 3$)$. Multiple regression results in Table 4 further showed that while psychological control, parental expectations, and parental discipline were significant predictors of Chinese parental control measures, parental knowledge and parental monitoring either had very weak or no influence on Chinese parental control.

\section{Research Question 3 (Hypotheses 2A and 2B)}

Regarding the relationships between Chinese parental control measures and indices of parent-child relational qualities, results showed that the related linkages were weak (Table 5). In fact, correlation coefficients on the linkages between these two scales and parent-child relational quality measures were significantly lower than those relationships between Western measures of parental control (parental monitoring and parental knowledge) and parent-child relational qualities, thus supporting Hypothesis 2A. Nevertheless, the relationships between these two domains appeared to be moderated by the degree of psychological control. As shown in Table 6, for those who perceived parental control to be low, there was a positive relationship between parental control based on Chinese concepts and parent-child relational qualities. The findings give support to Hypothesis $2 \mathrm{~B}$.

Furthermore, partial correlation analyses removing the effect of Grade 7 paternal psychological control showed that Grade 7 CPCS scores were positively related to readiness to communicate with father $(\mathrm{r}=.10, \mathrm{p}<.0001)$, trust of father $(\mathrm{r}=.10, \mathrm{p}<.0001)$ and satisfaction with paternal control $(\mathrm{r}=.08, \mathrm{p}<.0001)$ at Grade 9. Similarly, partial correlation analyses removing the effect of Grade 7 maternal psychological control showed that Grade 7 CMCS scores were posi- 
Table 2. Frequency of Responses to the Items of the Chinese Paternal Control Scale (CPCS) and Chinese Maternal Control Scale (CMCS)

\begin{tabular}{|c|c|c|c|c|c|c|}
\hline \multirow{2}{*}{ Item } & \multicolumn{6}{|c|}{$\begin{array}{l}\text { Cumulative Percentage of Responses to "Agree" and } \\
\text { "Strongly Agree" Response Options }\end{array}$} \\
\hline & \multicolumn{2}{|c|}{ Grade 7} & \multicolumn{2}{|c|}{ Grade 8} & \multicolumn{2}{|c|}{ Grade 9} \\
\hline 1. My father expects me to be mature (sheng xing). & 86.4 & 91.9 & 90.6 & 95.2 & 91.0 & 95.3 \\
\hline 2. My father expects me to be obedient ("guai" and "ting hua"). & 90.0 & 94.1 & 91.9 & 96.0 & 91.7 & 94.7 \\
\hline $\begin{array}{l}\text { 4. My father expects me to have good behavior so that I will not bring dishonor to the } \\
\text { family ("you ru jia sheng"). }\end{array}$ & 51.4 & 53.5 & 52.0 & 54.8 & 51.1 & 53.8 \\
\hline $\begin{array}{l}\text { 5. My father expects me to have good behavior so that he will not be criticized by others } \\
\text { as having no family teaching ("wu jia jiao"). }\end{array}$ & 47.6 & 41.2 & 58.6 & 62.5 & 56.0 & 61.0 \\
\hline 6. My father expects me to respect him. & 73.0 & 78.6 & 75.4 & 80.0 & 75.5 & 80.0 \\
\hline 7. My father is very harsh in his discipline. & 59.7 & 47.1 & 36.5 & 44.9 & 34.3 & 40.6 \\
\hline 11. When I don't meet my father's expectation, he urges me ("du cu”) to work hard. & 75.5 & 85.3 & 73.1 & 84.7 & 72.6 & 83.0 \\
\hline $\begin{array}{l}\text { 12. My father expects me to interact with older people ("chang bei") with respect and } \\
\text { courtesy. }\end{array}$ & 94.2 & 96.9 & 93.4 & 96.0 & 92.1 & 95.1 \\
\hline
\end{tabular}

Note: The cumulative percentage figure is computed by adding the percentage of responses to the "Agree" and "Strongly Agree" response options.

tively related to readiness to communicate with mother $(\mathrm{r}=$ $.09, \mathrm{p}<.0001)$, trust of mother $(\mathrm{r}=.10, \mathrm{p}<.0001)$ and satisfaction with maternal control $(\mathrm{r}=.06, \mathrm{p}<.01)$ at Grade 9. However, it is noteworthy that the effect size of the partial correlation coefficients was not high.

\section{DISCUSSION}

\section{Reliability of CPCS and CMCS}

This paper examines Chinese parental control characteristics and its correlates. As far as the reliability of the CPCS and CMCS is concerned, results showed that both scales are internally consistent. The development of the CPCS and CMCS and accumulation of research findings on the psychometric properties of these scales enable family therapists and allied professionals to assess Chinese parental control in the Chinese culture in an objective manner. Because there are few instruments assessing Chinese family processes (Shek, 2006a), the present study can be regarded as a constructive response.

Of course, one can query whether these items really measure parental control based on indigenous Chinese parenting concepts. There are three responses to this query. First, the items in these scales were developed after a thorough review of the literature on Chinese parenting (e.g., family handbooks). Second, a psychologist with substantial experience in scale construction and an experienced social worker were involved in the scale development process. Finally, Shek (2007) provided support for the construct validity of the scale.

\section{Research Question 1: Profiles of Perceived Chinese Pa- rental Control}

Several observations can be highlighted from the responses to the items in the CPCS and CMCS (Table 1). First, although parents were perceived to have expectations about their children (items 1, 2, 3, 6 and 12), they were not perceived to have high expectations about their children's good behavior because of family reasons (items 4 and 5). This observation suggests that although traditional parental expectations are still present, parental expectations based on family name and honor are weakening. Second, the findings suggest that contemporary parents in Hong Kong do not place strong emphases on teaching and supervision of their children (item 9 and item 10). Third, the findings suggest that harsh parental discipline gradually weakens in contemporary Chinese culture. Consistent with other research findings, these observations indicate that traditional Chinese parenting characteristics such as harsh punishment are gradually fading (Ho, 1986). Shek (in press-b) showed that the traditional Chinese parenting pattern of "strict fathers, kind mothers" gradually changed to "strict mothers, kind fathers". Shek (in press-a) also showed that although parental psychological control was high in contemporary Chinese parents, parental behavioral control (such as monitoring and knowledge) was not high.

The normative profile of perceived Chinese parental control clarifies our understanding of the nature of Chinese parental control perceived by early adolescents in Hong Kong. Basically, the findings point out the paradoxical nature of Chinese parental control - a mixture of high parental expectations with relatively loose parental monitoring and discipline. In the context of family intervention, parents could be helped to examine their parental control characteristics to see whether parental behavioral control should be strengthened (Shek, in press-b). The strong parental expectations on maturity, obedience, virtues, and respect for parents and old people may also help family workers to understand the origin of inter-generational conflicts in the family. On a macro level, the present findings provide some useful pointers for policy makers to consider what parenting characteristics 
should be promoted in Hong Kong. For example, the percentages of responses to item 4 and item 5 suggest that there may be a need to strengthen family teaching ("jia jiao") among Chinese families in Hong Kong. The present findings also suggest that parents should promote self-reflection in their children (item 9).

Table 3. Relationships Between Chinese Parental Control Scales (CPCS and CMCS) and Measures of Perceived Psychological Control, Behavioral Control and Parental Endorsement of Traditional Chinese Parenting Concepts

\begin{tabular}{|c|c|c|c|c|c|c|}
\hline \multicolumn{7}{|c|}{ Grade 7} \\
\hline \multirow{2}{*}{ CPCS } & PPSY & PEXP & PDIS & PKNO & PMON & PBEF \\
\hline & $.37 *$ & $.51^{*}$ & $.50 *$ & $.17 *$ & $.23 *$ & $.54 *$ \\
\hline \multirow{2}{*}{ CMCS } & MPSY & MEXP & MDIS & MKNO & MMON & MBEF \\
\hline & $.34 *$ & $.49 *$ & $.51 *$ & $.17 *$ & $.25^{*}$ & $.55^{*}$ \\
\hline \multicolumn{7}{|c|}{ Grade 8} \\
\hline \multirow{2}{*}{ CPCS } & PPSY & PEXP & PDIS & PKNO & PMON & PBEF \\
\hline & $.40 *$ & $.49^{*}$ & $.49^{*}$ & $.18^{*}$ & $.23^{*}$ & $.55^{*}$ \\
\hline \multirow{2}{*}{ CMCS } & MPSY & MEXP & MDIS & MKNO & MMON & MBEF \\
\hline & $.40 *$ & $.55^{*}$ & $.48 *$ & $.19^{*}$ & $.33^{*}$ & $.57 *$ \\
\hline \multicolumn{7}{|c|}{ Grade 9} \\
\hline \multirow{2}{*}{ CPCS } & PPSY & PEXP & PDIS & PKNO & PMON & PBEF \\
\hline & $.41 *$ & $.52 *$ & $.47^{*}$ & $.12 *$ & $.21 *$ & $.56^{*}$ \\
\hline \multirow{2}{*}{ CMCS } & MPSY & MEXP & MDIS & MKNO & MMON & MBEF \\
\hline & $.39 *$ & $.55^{*}$ & $.48^{*}$ & $.16^{*}$ & $.30 *$ & $.61 *$ \\
\hline
\end{tabular}

Note. CPCS: Chinese Paternal Control Scale. PPSY: Paternal Psychological Control Scale. PEXP: Paternal Expectation Scale. PDIS: Paternal Discipline Scale. PKNO: Paternal Knowledge Scale. PMON: Paternal Monitoring Scale. PBEF: Paternal Parenting Beliefs Scale. CMCS: Chinese Maternal Control Scale. MPSY: Maternal Psychological Control Scale. MEXP: Maternal Expectation Scale. MDIS: Maternal Discipline Scale. MKNO: Maternal Knowledge Scale. MMON: Maternal Monitoring Scale. MBEF: Maternal Parenting Beliefs Scale.

A two-tailed multistage Bonferroni procedure was used to analyze the data at each grade. $p F W$ is based on the familywise Type 1 error rate. $p T$ is the Type 1 error rate per test.

All correlation coefficients on the linkages between Chinese parental control measures (CPCS and CMCS) and psychological control, parental expectation and parental discipline were significantly higher than the related coefficients based on parental knowledge and parental monitoring ( $<<.05$ in all cases).

$* p F W<.01 ; p T<.0003$.

\section{Research Question 2 (Research Hypotheses 1A, 1B and 1C)}

The present findings showed that the CPCS and CMCS scores were significantly correlated with measures of parental psychological control, behavioral control and parental endorsement of traditional Chinese cultural beliefs (Hypothesis 1A and Hypothesis 1B). However, the linkages between Chinese parental control and psychological control, parental expectation and parental discipline were stronger than the relationships between Chinese parental control and parental monitoring and parental knowledge (Hypothesis 1C). The present study basically replicated the research findings reported by Shek (2007) over a period of three years.
Table 4. Predictors of Chinese Parental Control Measures at Grade 7, Grade 8 and Grade 9

\begin{tabular}{|c|c|c|c|}
\hline \multirow{2}{*}{ Grade 7 DV } & \multicolumn{3}{|c|}{ Total Sample } \\
\hline & Predictors & Beta & $\mathrm{R}^{2}$ \\
\hline \multirow[t]{5}{*}{ CPCS } & PPSY & $.27 * * *$ & .40 \\
\hline & PEXP & $.30 * * *$ & \\
\hline & PDIS & $.30 * * *$ & \\
\hline & PKNO & $-.03 n s$ & \\
\hline & PMON & $-.01 \mathrm{~ns}$ & \\
\hline \multirow[t]{5}{*}{$\mathrm{CMCS}$} & MPSY & $.22 * * *$ & .39 \\
\hline & MEXP & $.28 * * *$ & \\
\hline & MDIS & $.34 * * *$ & \\
\hline & MKNO & $-.01 \mathrm{~ns}$ & \\
\hline & MMON & $.01 \mathrm{~ns}$ & \\
\hline \multirow{2}{*}{ Grade 8 DV } & \multicolumn{3}{|c|}{ Total Sample } \\
\hline & Predictors & Beta & $\mathrm{R}^{2}$ \\
\hline \multirow[t]{5}{*}{$\mathrm{CPCS}$} & PPSY & $.31 * * *$ & .40 \\
\hline & PEXP & $.30 * * *$ & \\
\hline & PDIS & $.28 * * *$ & \\
\hline & PKNO & $.00 \mathrm{~ns}$ & \\
\hline & PMON & $-.03 \mathrm{~ns}$ & \\
\hline \multirow[t]{5}{*}{ CMCS } & MPSY & $.29 * * *$ & .43 \\
\hline & MEXP & $.32 * * *$ & \\
\hline & MDIS & $.24 * * *$ & \\
\hline & MKNO & $-.01 \mathrm{~ns}$ & \\
\hline & MMON & $.07 *$ & \\
\hline \multirow{2}{*}{$\begin{array}{c}\text { Grade } 9 \\
\text { DV }\end{array}$} & \multicolumn{3}{|c|}{ Total Sample } \\
\hline & Predictors & Beta & $\mathrm{R}^{2}$ \\
\hline \multirow[t]{5}{*}{ CPCS } & PPSY & $.29 * * *$ & .42 \\
\hline & PEXP & $.37 * * *$ & \\
\hline & PDIS & $.27 * * *$ & \\
\hline & PKNO & $-.07 *$ & \\
\hline & PMON & $-.04 n s$ & \\
\hline \multirow[t]{5}{*}{ CMCS } & MPSY & $.27 * * *$ & .42 \\
\hline & MEXP & $.35 * * *$ & \\
\hline & MDIS & $.24 * * *$ & \\
\hline & MKNO & $.01 \mathrm{~ns}$ & \\
\hline & MMON & $.03 \mathrm{~ns}$ & \\
\hline
\end{tabular}

Note. CPCS: Chinese Paternal Control Scale. PPSY: Paternal Psychological Control Scale. PEXP: Paternal Expectation Scale. PDIS: Paternal Discipline Scale. PKNO: Paternal Knowledge Scale. PMON: Paternal Monitoring Scale. CMCS: Chinese Maternal Control Scale. MPSY: Maternal Psychological Control Scale. MEXP: Maternal Expectation Scale. MDIS: Maternal Discipline Scale. MKNO: Maternal Knowledge Scale. MMON: Maternal Monitoring Scale.

$* * * \mathrm{p}<.001 ; * * \mathrm{p}<.01 ; * \mathrm{p}<.05 ;$ ns non-significant 
Table 5. Relationships Between Measures of Perceived Parental Control and Parent-Child Relational Qualities

\begin{tabular}{|c|c|c|c|c|c|c|}
\hline Grade 7 & PPSY & PEXP & PDIS & PKNO & PMON & $\mathrm{CPCS}$ \\
\hline FTRU & $-.48 *$ & $.09^{*}$ & $.13 *$ & $.41^{*}$ & $.27 *$ & $-.07 *$ \\
\hline TRUF & $-.50 *$ & $.18^{*}$ & $.22 *$ & $.46^{*}$ & $.36^{*}$ & $-.02 \mathrm{~ns}$ \\
\hline RCF & $-.31 *$ & $.27^{*}$ & $.28^{*}$ & $.54 *$ & $.52 *$ & $.06^{*}$ \\
\hline SATF & $-.48 *$ & $.26^{*}$ & $.28 *$ & $.51^{*}$ & $.40^{*}$ & $.04 \mathrm{~ns}$ \\
\hline \multicolumn{7}{|l|}{ Grade 8} \\
\hline FTRU & $-.52 *$ & $.11^{*}$ & $.12 *$ & $.41^{*}$ & $.30 *$ & $-.08^{*}$ \\
\hline TRUF & $-.51 *$ & $.24 *$ & $.27^{*}$ & $.51 *$ & $.40^{*}$ & $-.00 \mathrm{~ns}$ \\
\hline $\mathrm{RCF}$ & $-.33 *$ & $.28^{*}$ & $.29^{*}$ & $.57 *$ & $.53 *$ & $.06^{*}$ \\
\hline SATF & $-.53^{*}$ & $.24^{*}$ & $.28^{*}$ & $.52 *$ & $.40^{*}$ & $-.02 \mathrm{~ns}$ \\
\hline \multicolumn{7}{|l|}{ Grade 9} \\
\hline FTRU & $-.55^{*}$ & $.03 \mathrm{~ns}$ & $.11 *$ & $.37 *$ & $.23 *$ & $-.12 *$ \\
\hline TRUF & $-.49 *$ & $.17^{*}$ & $.27 *$ & $.47 *$ & $.38^{*}$ & $-.03 \mathrm{~ns}$ \\
\hline $\mathrm{RCF}$ & $-.32 *$ & $.22 *$ & $.32 *$ & $.58 *$ & $.51^{*}$ & $.01 \mathrm{~ns}$ \\
\hline SATF & $-.53^{*}$ & $.17^{*}$ & $.28^{*}$ & $.50 *$ & $.38^{*}$ & $-.04 \mathrm{~ns}$ \\
\hline Grade 7 & MPSY & MEXP & MDIS & MKNO & MMON & CMCS \\
\hline MTRU & $-.52 *$ & $.03 \mathrm{~ns}$ & $.11 *$ & $.40 *$ & $.27 *$ & $-.06^{*}$ \\
\hline TRUM & $-.53 *$ & $.10^{*}$ & $.16^{*}$ & $.45^{*}$ & $.35^{*}$ & $-.04 \mathrm{~ns}$ \\
\hline $\mathrm{RCM}$ & $-.35^{*}$ & $.18^{*}$ & $.23^{*}$ & $.54 *$ & $.46^{*}$ & $.05 \mathrm{~ns}$ \\
\hline SATM & $-.50 *$ & $.14 *$ & $.23 *$ & $.49 *$ & $.38^{*}$ & $.02 \mathrm{~ns}$ \\
\hline \multicolumn{7}{|l|}{ Grade 8} \\
\hline MTRU & $-.49 *$ & $.00 \mathrm{~ns}$ & $.12 *$ & $.38^{*}$ & $.24 *$ & $-.08^{*}$ \\
\hline TRUM & $-.49 *$ & $.10^{*}$ & $.18^{*}$ & $.45^{*}$ & $.34 *$ & $-.02 \mathrm{~ns}$ \\
\hline $\mathrm{RCM}$ & $-.34 *$ & $.17^{*}$ & $.22 *$ & $.51 *$ & $.41^{*}$ & $.03 \mathrm{~ns}$ \\
\hline SATM & $-.49 *$ & $.14^{*}$ & $.23 *$ & $.49^{*}$ & $.34 *$ & $.01 \mathrm{~ns}$ \\
\hline \multicolumn{7}{|l|}{ Grade 9} \\
\hline MTRU & $-.53 *$ & $-.06^{*}$ & $.08^{*}$ & $.38 *$ & $.17^{*}$ & $-.07 *$ \\
\hline TRUM & $-.53 *$ & $.02 \mathrm{~ns}$ & $.16^{*}$ & $.44 *$ & $.30 *$ & $-.05^{*}$ \\
\hline $\mathrm{RCM}$ & $-.33^{*}$ & $.11^{*}$ & $.21^{*}$ & $.53 *$ & $.37^{*}$ & $.02 \mathrm{~ns}$ \\
\hline SATM & $-.54 *$ & $.03 \mathrm{~ns}$ & $.17 *$ & $.46^{*}$ & $.28^{*}$ & $-.06^{*}$ \\
\hline
\end{tabular}

Note. PPSY: Paternal Psychological Control Scale. PEXP: Paternal Expectation Scale. PDIS: Paternal Discipline Scale. PKNO: Paternal Knowledge Scale. PMON: Paternal Monitoring Scale. CPCS: Chinese Paternal Control Scale. FTRU: Paternal Trust of Children Scale. TRUF: Children's Trust of Father Scale. SATF: Satisfaction with Paternal Control Scale. RCF: Readiness to Communicate with Father Scale. MPSY: Maternal Psychological Control Scale. MEXP: Maternal Expectation Scale. MDIS: Maternal Discipline Scale. MKNO: Maternal Knowledge Scale. MMON: Maternal Monitoring Scale. CMCS: Chinese Maternal Control Scale. MTRU: Maternal Trust of Children Scale. TRUM: Children's Trust of Mother Scale. SATM: Satisfaction with Maternal Control Scale. RCM: Readiness to Communicate with Mother Scale.

A two-tailed multistage Bonferroni procedure was used to analyze the data (144 correlation coefficients). $p F W$ is based on the familywise Type 1 error rate. $p T$ is the Type 1 error rate per test.

All correlation coefficients between CPCS (and CMCS) and measures of parent-child relational qualities were significantly lower than the related correlation coefficients based on parental monitoring and parental knowledge as measures of parental control $(\mathrm{p}<.05$ in all cases).

$* p F W<.05 ; p T<.002$.
This observation suggests that Chinese parental control is less related to parental behavioral control in terms of monitoring and knowledge.

Besides contributing to the limited scientific literature on Chinese families (Shek, 2006b), the present findings can help family researchers construct models on Chinese parenting processes. Although models on parental control have been constructed in the West (e.g., Becker, 1964; Schaefer, 1959) and most of the existing studies of Chinese parenting are based on Western models, indigenous Chinese parenting models are almost non-existent. The present findings suggest that traditional Chinese parental control is characterized by psychological control, expectations, discipline and traditional Chinese beliefs which are weakly related to parental monitoring and parental knowledge. This conglomeration of different attributes of parenting is quite interesting and it cannot be adequately accommodated within existing Western models on parenting.

\section{Research Question 3 (Hypotheses 2A and 2B)}

In contrast to Western measures of parental control, the findings revealed that both CPCS and CMCS had only weak relationships to different measures of parent-child relational qualities. Compared with Western measures of parental control (parental monitoring and parental knowledge), Chinese parental control measures had a much weaker relationship to parent-child relational qualities (Hypothesis 2A). This observation suggests that Chinese parental control indexed by indigenous Chinese parenting concepts is conceptually distinct from Western parental control concepts. Again, the present findings underscore the uniqueness of Chinese parental control indexed by indigenous Chinese parenting concepts.

The findings further suggest that psychological control is a factor that moderates the relationships between Chinese parental control measures and parent-child relational qualities (Hypothesis 2B). The observation that Chinese parental control characteristics were positively related to parent-child relational qualities under low but not under high psychological control condition obviously challenges the common view that strict Chinese parental control attributes are detrimental to parent-child relationship. The partial correlation analyses further showed that Chinese parental control was positively related to parent-child relational qualities after the effect of psychological control had been removed. The present study clearly suggests that the relationship between Chinese parental control and parent-child relational quality is not simple. Practically, family practitioners including family therapists and social workers should be sensitive to the role played by psychological control in analyzing Chinese parental control. In handling cases with parent-adolescent conflict, it is suggested that family practitioners should examine psychological control of the parents. With reference to the comment of Barber (1996) that "although psychological control was included in some of the earliest conceptualization of parenting and continues to be implicit in much of the major work, focused attention to the construct has been lacking" (p.3298), more research should be conducted to examine psychological control in Chinese parents. 
Table 6. Relationship Between Chinese Perceived Parental Control Measures and Parent-Child Relational Quality Under High and Low Levels of Parental Psychological Control

\begin{tabular}{|c|c|c|c|c|c|}
\hline Control Measure & Psychological Control (Moderator) & FTRU/MTRU & TRUF/TRUM & SATF/SATM & $\mathrm{RCF} / \mathrm{RCM}$ \\
\hline Grade 7 & High PPSY & $-.00 \mathrm{~ns}$ & $.00 \mathrm{~ns}$ & $.01 \mathrm{~ns}$ & $.05 \mathrm{~ns}$ \\
\hline Grade 7 & High MPSY & $-.01 \mathrm{~ns}$ & $-.02 \mathrm{~ns}$ & $.06 \mathrm{~ns}$ & $.04 \mathrm{~ns}$ \\
\hline CMCS & Low MPSY & $.13 *$ & $.18^{*}$ & $.24 *$ & $.25 *$ \\
\hline $\mathrm{CPCS}$ & Low PPSY & $.14^{*}$ & $.26^{*}$ & $.29 *$ & $.28 *$ \\
\hline Grade 8 & High MPSY & $-.05 \mathrm{~ns}$ & $-.01 \mathrm{~ns}$ & $.02 \mathrm{~ns}$ & $.01 \mathrm{~ns}$ \\
\hline CMCS & Low MPSY & $.11 *$ & $.23^{*}$ & $.27 *$ & $.23 *$ \\
\hline Grade 9 & High PPSY & $-.15^{*}$ & $-.07 \mathrm{~ns}$ & $-.09 \mathrm{~ns}$ & $-.03 \mathrm{~ns}$ \\
\hline
\end{tabular}

Note. CPCS: Chinese Paternal Control Scale. PPSY: Chinese Paternal Psychological Control Scale. FTRU: Paternal Trust of Children Scale. TRUF: Children's Trust of Father Scale. SATF: Satisfaction with Paternal Control Scale. RCF: Readiness to Communicate with Father Scale. CMCS: Chinese Maternal Control Scale. MPSY: Chinese Maternal Psychological Control Scale. MTRU: Maternal Trust of Children Scale. TRUM: Children's Trust of Mother Scale. SATM: Satisfaction with Maternal Control Scale. RCM: Readiness to Communicate with Mother Scale.

A two-tailed multistage Bonferroni procedure was used to analyze the data in each sample. $p F W$ is based on the familywise Type 1 error rate. $p T$ is the Type 1 error rate per test. $* p F W<.05 ; p T<.002$ for the samples with high parental psychological control ( 24 correlation coefficients).

$* p F W<.05 ; p T<.002$ for the samples with low parental psychological control (24 correlation coefficients).

\section{Limitations and Future Research Directions}

There are several limitations of this study. First, because the assessment of perceived parental control measures was based on self-report measures from the perspective of the child, there might be bias involved. The inclusion of observational data in real life settings based on the outsiders and collection of data based on other members within the family would give a more complete picture as far as assessment of the related processes is concerned. Second, because the sample was recruited from Chinese adolescents in Hong Kong, there is a need to replicate the findings in different Chinese contexts. Third, because the focus of the study was on early adolescence, it would be exciting if future studies based on late adolescents could be carried out. Fourth, further analyses based on linear mixed models and latent variables analyses should be carried out which could take sufficient advantage of the longitudinal data. Fifth, it is noteworthy that the effect size of the prospective partial correlation coefficients was on the low side. Finally, the inclusion of other indigenous concepts of parental control (e.g., Chao, 1994) in future would give a better picture regarding the nature of parental control in Hong Kong. Despite these limitations, the present study constitutes a pioneering longitudinal attempt in examining Chinese parental control in the realm of Chinese family research.

This work was financially supported by the Research Grants Council of the Government of the Hong Kong Special Administrative Region, Hong Kong (Grant CUHK4293/03H) and Wofoo Foundation.

\section{REFERENCES}

Barber, B. K. (1996). Parental psychological control: Revisiting a neglected construct. Child Development, 67, 3296-3319.
Barber, B. K. (Ed.). (2002). Intrusive parenting: How psychological control affects children and adolescents. Washington, DC: American Psychological Association.

Barber, B. K., \& Harmon, E. L. (2002). Violating the self: Parental psychological control of children and adolescent. In B. K. Barber (Ed.), Intrusive parenting: How psychological control affects children and adolescents (pp.15-52). Washington, DC: American Psychological Association.

Becker, W. C. (1964). Consequences of different kinds of parental discipline. In M. L. Hoffman and L. W. Hoffman (Eds.), Review of child development research (pp.169-208). New York: Russell Sage Foundation.

Chao, R. K. (1994). Beyond parental control and authoritarian parenting style: Understanding Chinese parenting through the cultural notion of training. Child Development, 65, 1111-1119.

Chao, R. K., \& Tseng, V. (2002). Parenting of Asians. In M. H. Bornstein (Ed.), Handbook of parenting Vol. 4 (pp.59-93). Mahwah, NJ: Erlbaum.

Galambos, N. L., Barker, E. T., \& Almeida, D. M. (2003). Parents do matter: Trajectories of change in externalizing and internalizing problems in early adolescence. Child Development, 74, 578-594.

Harakeh, Z., Scholte, R., Vermulst, A. A., de Vries, H., \& Engels, R. (2004). Parental factors and adolescents' smoking behavior: An extension of the theory of planned behavior. Preventive Medicine, 39, 951-961.

Ho, D. Y. F. (1986). Chinese patterns of socialization: A critical review. In M. Bond (Ed.), The psychology of the Chinese people (pp.1-37). New York: Oxford University Press.

Kerr, M., \& Stattin, H. (2000). What parents know, how they know it, and several forms of adolescent adjustment: Further support for a reinterpretation of monitoring. Developmental Psychology, 36, 366380.

Larzelere, R. E., \& Mulaik, S. A. (1977). Single-sample tests for many correlations. Psychological Bulletin, 84, 557-569.

Pettit, G. S., Laird, R. D., Dodge, K. A., Bates, J. E., \& Criss, M. M. (2001). Antecedents and behavior-problem outcomes of parental monitoring and psychological control in early adolescence. Child Development, 72, 583-598.

Schaefer, E. S. (1959). A circumplex model for maternal behavior. Journal of Abnormal and Social Psychology, 59, 226-235.

Shek, D. T. L. (2005). Perceived parental control processes, parent-child relational qualities, and psychological well-being in Chinese ado- 
lescents with and without economic disadvantage. Journal of Genetic Psychology, 166, 171-188.

Shek, D. T. L. (2006a). Assessment of perceived parental psychological control in Chinese adolescents in Hong Kong. Research on Social Work Practice, 16, 382-391.

Shek, D. T. L. (2006b). Chinese family research: Puzzles, progress, paradigms, and policy implications. Journal of Family Issues, 27(3), 275-284.

Shek, D. T. L. (2006c). Perceived parental behavioral control and psychological control in Chinese adolescents in Hong Kong. American Journal of Family Therapy, 34, 163-176.

Shek, D. T. L. (2007). Perceived parental control based on indigenous Chinese parental control concepts in adolescents in Hong Kong. American Journal of Family Therapy, 35, 123-137.

Shek, D. T. L. Perceived parental control and parent-child relational qualities in early adolescents in Hong Kong: Parent gender, child gender and grade differences. Sex Roles, (in press-a).

Shek, D. T. L. Parent-adolescent trust and parent-adolescent relationship in Chinese families in Hong Kong: Implications for parent education and family therapy. American Journal of Family Therapy, (in press-b).

Shek, D. T. L., \& Lai, M. F. (2000). Conceptions of an ideal family in Confucian thoughts: Implications for individual and family counseling. Asian Journal of Counseling, 7(2), 85-104.

Shek, D. T. L., \& Lee, T. Y. (2005). Perceived parental behavioral control, psychological control and parent-child relational qualities in Chinese adolescents in Hong Kong. In M. J. Kane (Ed.), Contemporary issues in parenting (pp.47-63). New York: Nova Science Publishers.

Shek, D. T. L., Lee, T. Y., \& Chow, J. T. W. (2006). Perceived parental behavioral control, psychological control and parent-child relational qualities in Chinese adolescents in Hong Kong. Journal of Youth Studies, 9, 138-155. (In Chinese.)

Smetana, J. G., \& Daddis, C. (2002). Domain-specific antecedents of parental psychological control and monitoring: The role of parenting beliefs and practices. Child Development, 73, 563-580.

Yang, K. S. (1981). The formation and change of Chinese personality: A cultural-ecological perspective. Acta Psychologica Taiwanica, 23, $39-55$.

Received: February 22, 2008

Revised: March 13, 2008

Accepted: April 7, 2008

(C) Daniel T.L. Shek; Licensee Bentham Open.

This is an open access article distributed under the terms of the Creative Commons Attribution License (http://creativecommons.org/license/by/2.5/), which permits unrestrictive use, distribution, and reproduction in any medium, provided the original work is properly cited. 